\title{
Simulation of GaN-based Light-Emitting Devices
}

\author{
Joachim Piprek \\ Solid-State Lighting and Display Center \\ Materials Department, College of Engineering \\ University of California, Santa Barbara, CA 93106 \\ piprek@ieee.org
}

\begin{abstract}
Comprehensive physics-based simulations of GaN-based light-emitting diodes and laser diodes are presented. The talk discusses unique material properties of nitride compounds as well as performance-limiting physical mechanisms like self-heating, current crowding, and carrier leakage. Measurements are used to validate the models and to extract critical device parameters.
\end{abstract}

\section{Introduction}

GaN-based light-emitting devices were pioneered by Shuji Nakamura and coworkers in the 1990s [1] and they find increasing use in an array of applications, e.g., in traffic signals, full-color displays, and data storage systems. Wurtzite InGaN and AlGaN compounds are employed in these devices, which are able to cover a wide wavelength range (Fig. 1). InGaN quantum wells have been successfully used in blue or green light emitting diodes (LEDs), as well as in blue laser diodes (LDs). AlGaN quantum wells are currently of great interest for ultraviolet (UV) light generation within white light sources and other applications.

Despite intense research efforts worldwide on GaN-based devices, major technological challenges, as well as the need for a more detailed understanding of microscopic physical processes, remain. Numerical simulation can help to investigate those processes and to establish quantitative links between material properties and measured device performance. This paper reviews some of the unique material properties found in nitride compounds and it then describes self-consistent simulations of AlGaN LEDs and InGaN laser diodes.

\section{Nitride Material Properties}

Nitride semiconductors suffer from the high activation energy of the magnesium acceptor, which causes the hole concentration to be considerably smaller than the acceptor concentration. A magnesium activation energy of $170 \mathrm{meV}$ was reported for $\mathrm{GaN}$ [2], which is assumed to increase by $3 \mathrm{meV}$ per percent of $\mathrm{Al}$ for $\mathrm{AlGaN}$. In $n$-type material, the silicon donor activation energy is about $20 \mathrm{meV}$.

The measured electron mobility in GaN is near $900 \mathrm{~cm}^{2} / \mathrm{Vs}$ at low doping, and it drops by more than an order of magnitude with higher doping [3]. Monte Carlo simulations of various nitride alloys have been employed to establish analytical 


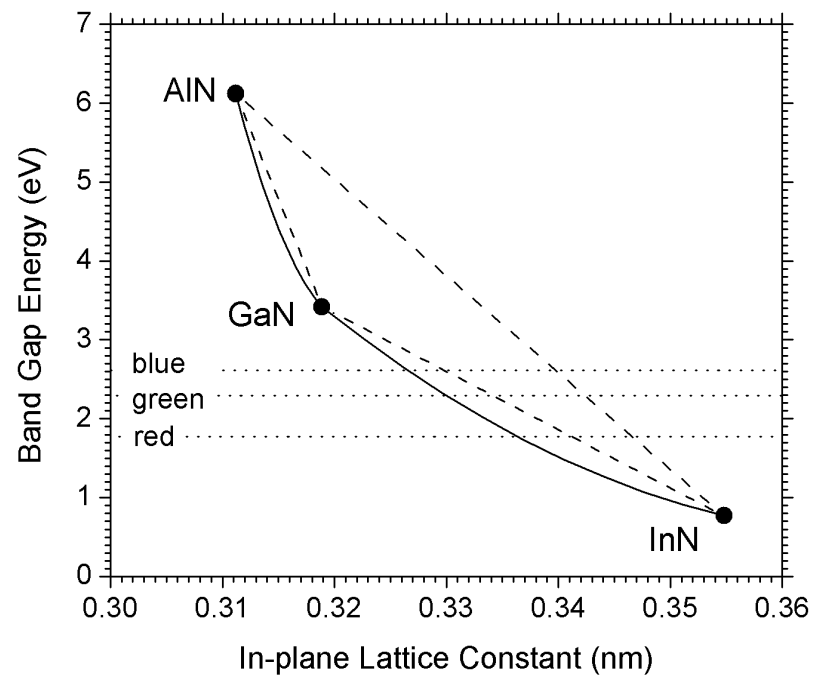

Figure 1: Energy band gap and lattice constant for wurtzite nitride compounds.

expressions for the electron mobility as a function of donor concentration, temperature, and electrostatic field [4]. Hole mobilities are much less investigated. For Mg-doped GaN, $\mu_{p}=15 \mathrm{~cm}^{2} / \mathrm{Vs}$ has been measured with low hole concentration and $2 \mathrm{~cm}^{2} / \mathrm{Vs}$ with high hole concentration [5]. This results in a hole conductivity that hardly varies with the doping density.

Built-in electrical fields in semiconductors can be caused by spontaneous polarization or by strain-induced polarization and were found to be much stronger in nitrides than in other III-V compounds [6, 7]. Since different alloy compositions show different polarization, net charges remain at their interfaces. The built-in fields significantly affect the properties of quantum wells. Figure 2 illustrates the corresponding deformation of the band diagram. The transition energy is reduced by the built-in field, leading to a red-shift of the emission wavelength. The wider the quantum well, the more separated the electrons and holes, and the smaller the optical gain and spontaneous emission. However, with increasing carrier injection into the quantum well, charge screening is expected to reduce polarization field effects.

\section{AlGaN/GaN Light-Emitting Diode}

This section gives the results of a comprehensive physics-based three-dimensional (3D) simulation of an ultraviolet LED. We here employ the software APSYS [8] which self-consistently combines the $3 \mathrm{D}$ simulation of carrier transport, selfheating, quantum well effects, and optical ray tracing. The transport model includes drift and diffusion of electrons and holes, Fermi statistics, built-in polarization and thermionic emission at hetero-interfaces, as well as spontaneous 


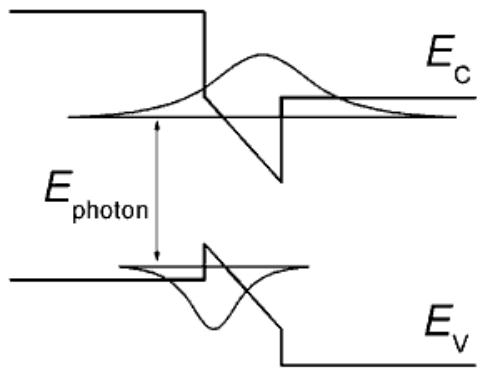

Figure 2: GaN-InGaN-GaN quantum well energy band diagram at $1 \mathrm{MV} / \mathrm{cm}$ polarization field with energy levels and wave functions.

and defect related Shockley-Read-Hall (SRH) recombination of carriers. For the quantum wells, Schrödinger and Poisson equations are solved iteratively in order to account for the quantum well deformation with changing device bias. Spontaneous emission of photons by electron-hole recombination within the quantum well is calculated using a free-carrier model and considering the detailed wurtzite valence band structure as well as a recent compilation of nitride bandstructure parameters [9]. Further details on models and material parameters can be found elsewhere [10].

The LED investigated was grown on $c$-face sapphire by metal organic chemical vapor deposition (MOCVD) [11]. The epitaxial structure includes a multiquantum well (MQW) active region with four $5 \mathrm{~nm}$ thick AlGaN quantum wells and a $p-\mathrm{Al}_{0.3} \mathrm{Ga}_{0.7} \mathrm{~N}$ electron blocker layer. $\mathrm{MQW}$ and blocker layer are sandwiched between two 42-period AlGaN superlattice (SL) cladding layers. A quadratic mesa with $300 \mu \mathrm{m}$ edge length is etched down to the $n$-GaN contact layer. The top $p$-contact layer is semi-transparent for top emission. The U-shaped $n$-side contact covers three of the four sides of the mesa. More details on device design, fabrication, and performance are given in [11, 12].

Figure 3 shows a $3 \mathrm{D}$ plot of the LED radiative recombination rate, which is strongest in the 4 quantum wells and which decays towards the device center, in agreement with experimental observations. This lateral non-uniformity is attributed to current crowding along the sides with an adjacent n-contact. The current density is highest in the two corners of the U-shaped contact. Ideally, electrons and holes meet in the quantum wells and recombine completely. How- 


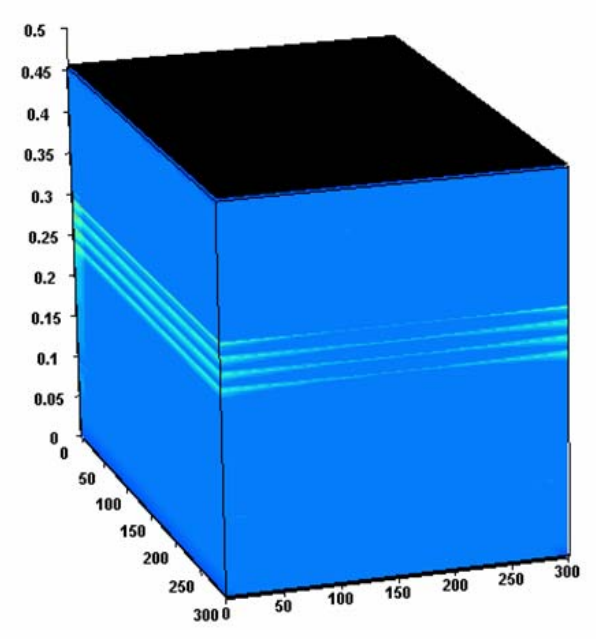

Figure 3: 3D plot of the LED radiative recombination rate.

ever, some holes leave the MQW region and leak into the lower n-cladding where they recombine with electrons. More severe is the electron leakage in the opposite direction. A large part of the electrons injected from the n-side into the MQW are found to leak into the upper p-cladding layer. In addition, the nonradiative SRH recombination within the quantum wells is about two orders of magnitude stronger than the radiative recombination, assuming a non-radiative carrier lifetime of $1 \mathrm{~ns}$. In other words, not more than $1 \%$ of the injected carriers contribute to the light emission.

Figure 4 shows the internal emission spectrum with and without built-in polarization. The polarization field leads to a shift of the quantum levels and to the creation of additional levels. The emission spectrum hardly shifts with increasing current, indicating little self-heating. Most photons are unable to escape from the LED, due to total internal reflection, mainly at the device surfaces, and to photon absorption, primarily in the bottom GaN layer. However,the main limitation of the emitted light power originates from the low internal quantum efficiency. Figure 5 plots the detected light power as function of injection current. The simulation is in good agreement with the measurement which indicates that model and parameters are fairly accurate in our simulation. Without builtin polarization, electrons and holes are less separated within each quantum well and the radiative emission rate is significantly enhanced, as shown by the dashed curve in Fig. 5. 


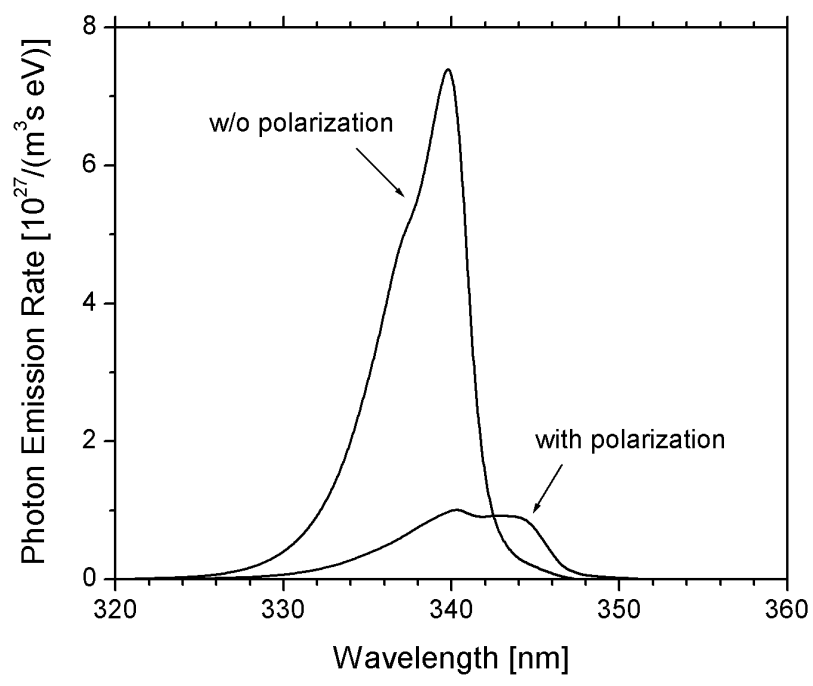

Figure 4: Internal spontaneous emission spectrum with and without built-in polarization at $50 \mathrm{~mA}$ injection current.

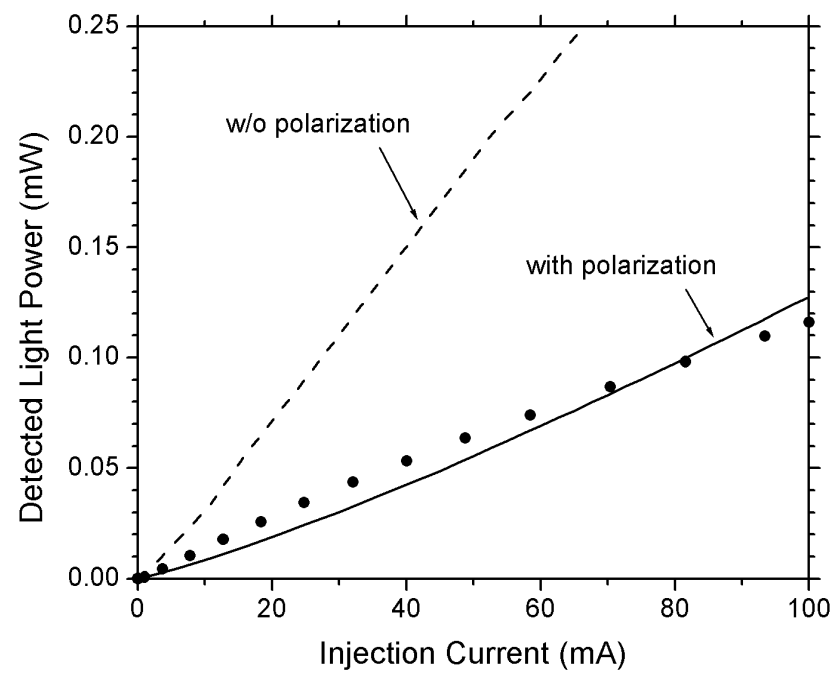

Figure 5: LED light vs. current characteristics (dots - measurement [11], lines simulation) 


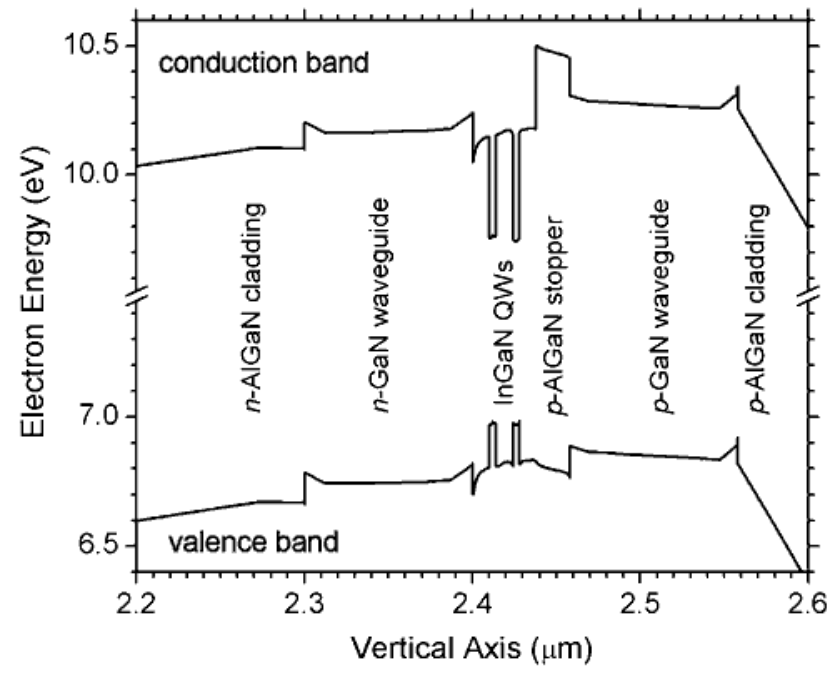

Figure 6: Energy band diagram of the InGaN/GaN laser diode at high injection.

\section{InGaN/GaN Laser Diode}

We here describe the 2D simulation of a high-power violet light emitting laser diode as demonstrated in [13]. The LASTIP laser simulator is used [14], which self-consistently combines wurtzite band structure and gain calculations with $2 \mathrm{D}$ simulations of wave guiding, carrier transport, and heat flux. More details on models and parameters can be found in [15].

The energy band diagram is shown in Fig. 6. The active region comprises two 4 -nm-thick InGaN quantum wells with $1.6 \%$ compressive strain as well as an $\mathrm{AlGaN}$ electron stopper layer. It is sandwiched between GaN separate confinement layers and superlattice cladding layers. In the simulation, we replace the superlattice by bulk AlGaN of average composition. For lateral confinement, a 3 - $\mu \mathrm{m}$-wide ridge waveguide structure is employed.

The optical gain mechanism in InGaN quantum wells is not yet fully understood. It may be strongly affected by a nonuniform Indium distribution. Internal polarization fields separate quantum confined electrons and holes, thereby reducing optical gain and spontaneous emission. However, screening by electrons and holes is expected to suppress quantum well polarization fields at high power operation. Therefore, we here assume rectangular quantum wells, i.e., polarization effects are neglected. We employ a simple free-carrier gain model including a Lorentzian broadening function with 0.1 ps scattering time [16].

In order to find agreement with the measured light-current characteristic, we use three main fit parameters: the internal modal loss $\alpha_{\mathrm{i}}$, the SRH recombination lifetime within the quantum wells $\tau_{\mathrm{qw}}$, and the thermal resistance $R_{\mathrm{th}}$. The modal loss controls the slope efficiency, the lifetime affects the threshold current, 


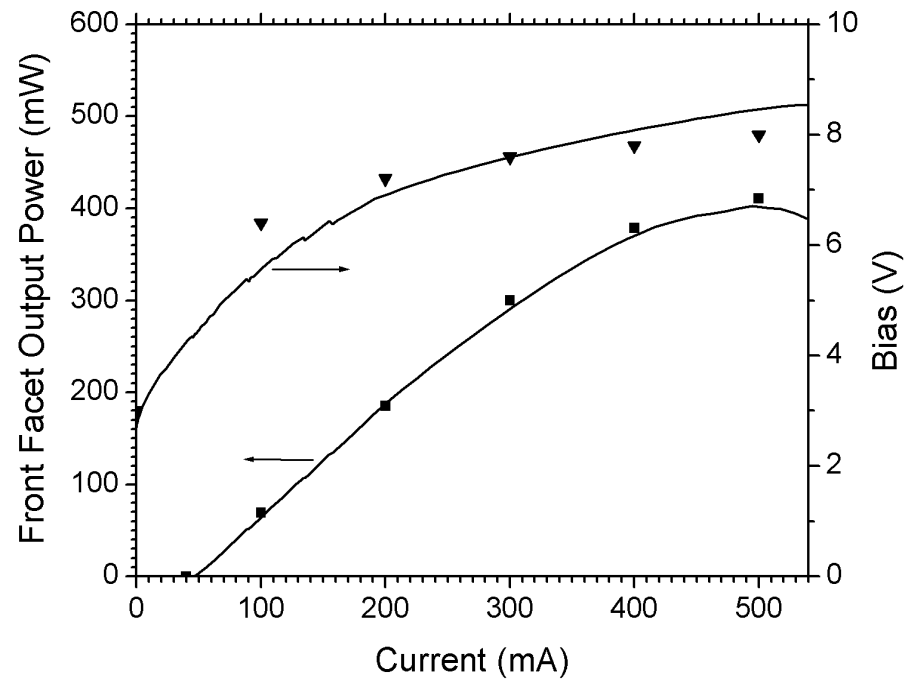

Figure 7: Current-voltage and light-current characteristics in continuous-wave operation: comparison of simulation (lines) and measurement (dots) [13].

and the thermal resistance has main impact on the power roll-off. The fitted values $\alpha_{\mathrm{i}}=12 \mathrm{~cm}^{-1}, \tau_{\mathrm{qw}}=0.5 \mathrm{~ns}$, and $R_{\mathrm{th}}=75 \mathrm{~K} / \mathrm{W}$ result in an excellent reproduction of the experimental curve (Fig. 7). All three numbers are reasonable and confirm the accuracy of the laser model.

Strong self-heating reduces the optical gain substantially, which is mainly caused by a wider Fermi spreading of carriers. Consequently, the carrier concentration increases with higher temperature. This leads to enhanced recombination losses but, most of all, to an escalation of electron leakage from the quantum wells into the $p$-side. This vertical electron leakage amounts to more than one-third of the total carrier losses at maximum power. As it prevents carriers from stimulated recombination, it is the main reason for the power roll-off.

\section{Summary}

This presentation has reviewed the current effort to achieve realistic simulations of GaN-based light emitters. Uncertain material properties are the main challenge with nitride devices. However, good agreement with measurements can be obtained by careful calibration of key parameters. 


\section{References}

[1] S. Nakamura, S. Pearton, and G. Fasol, The Blue Laser Diode. Berlin: SpringerVerlag, 2000.

[2] W. Götz, N. M. Johnson, J. Walker, D. P. Bour, and R. A. Street, "Activation of acceptors in Mg-doped GaN grown by metalorganic chemical vapor deposition," Appl. Phys. Lett., vol. 68, pp. 667-669, 1996.

[3] S. Nakamura, T. Mukai, and M. Senoh, "In situ monitoring and Hall measurements of GaN grown with GaN buffer layers," J. Appl. Phys., vol. 71, pp. 5543$5549,1992$.

[4] M. Farahmand, C. Garetto, E. Bellotti, K. F. Brennan, M. Goano, E. Ghillino, G. Ghione, J. D. Albrecht, and P. P. Ruden, "Monte Carlo simulation of electron transport in the III-nitride wurtzite phase material system: binaries and ternaries," J. Appl. Phys., vol. 48, pp. 535-542, 2001.

[5] S. Nakamura, M. Senoh, and T. Mukai, "Highly p-type Mg-doped GaN films grown with GaN buffer layers," Japan. J. Appl. Phys., Part 2, vol. 30, pp. L1708L1711, 1991.

[6] F. Bernardini, V. Fiorentini, and D. Vanderbilt, "Spontaneous polarization and piezoelectric constants of III-V nitrides," Phys. Rev. B, vol. 56, pp. R10024R10027, 1997.

[7] V. Fiorentini, F. Bernardini, and O. Ambacher, "Evidence for nonlinear macroscopic polarization in III-V nitride alloy heterostructures," apl, vol. 80, pp. 1204$1206,2002$.

[8] APSYS by Crosslight Software, Inc.

[9] I. Vurgaftman and J. R. Meyer, "Band parameters for nitrogen-containing semiconductors," J. Appl. Phys., vol. 94, pp. 3675-3691, 2003.

[10] J. Piprek and S. Li, "GaN-based light-emitting diode," in Optoelectronic Devices - Advanced Simulation and Analysis (J. Piprek, ed.), New York: Springer, 2004.

[11] T. Katona, Development of Ultraviolet Nitride-based Light-Emitting Diodes. PhD thesis, University of California at Santa Barbara, 2003.

[12] J. Piprek, T. Katona, S. P. DenBaars, and S. Li, "3D simulation and analysis of UV AlGaN/GaN LEDs," in Light-Emitting Diodes: Research, Manufacturing and Applications VII, vol. 5366, (Bellingham), SPIE- The International Society for Optical Engineering, 2004.

[13] S. Nakamura, M. Senoh, S. I. Nagahama, N. Iwasa, T. Yamada, T. Matsushita, H. Kiyoku, Y. Sugimoto, T. Kozaki, I. Umemoto, M. Sano, and K. Chocho, "Violet InGaN/GaN/AlGaN-based laser diodes with an output power of $420 \mathrm{~mW}$," Japan. J. Appl. Phys., Part 2, vol. 37, pp. L627-L629, 1998.

[14] LASTIP by Crosslight Software, Inc.

[15] J. Piprek, Semiconductor Optoelectronic Devices - Introduction to Physics and Simulation. San Diego: Academic Press, 2003.

[16] S. L. Chuang, "Optical gain of strained wurtzite GaN quantum well lasers," IEEE J. Quantum Electron., vol. 32, no. 10, pp. 1791-1799, 1996. 